\title{
Factores asociados a letalidad en pacientes con infección de herida quirúrgica. Estudio de casos y controles
}

\section{Factors associated to lethality in patients with surgical site infection. A study of cases and controls}

\author{
Brayant Andrade-Méndez ${ }^{1}$ (D) , Jorge Andrés Ramos-Castañeda² (i) , María del Mar Ruiz-García ${ }^{3}$ (D), Edilberto Suaza- \\ Calderón ${ }^{4}$ iD
}

1. Universidad Surcolombiana. Neiva, Colombia. Correo: brayant.andrade@usco.edu.co - https://orcid.org/0000-0001-7165-9361

2. Universidad CES. Medellín, Colombia. Correo: ramos.jorge@uces.edu.co - https://orcid.org/0000-0003-3297-0012

3. Universidad Surcolombiana. Neiva, Colombia. Correo: mariaruiz996@gmail.com - https://orcid.org/0000-0003-4155-5953

4. Universidad Surcolombiana. Neiva, Colombia. Correo: edisuaza@gmail.com - https://orcid.org/0000-0003-0021-1919

Tipología: Artículo de investigación científica y tecnológica

Para citar este artículo: Andrade-Méndez B, Ramos-Castañeda JA, Ruiz-García M, Suaza-Calderón E. Factores asociados a letalidad en pacientes con infección de herida quirúrgica. Estudio de casos y controles. Duazary. 2019 enero;17(1): 26 - 35. Doi: http://dx.doi.org/10.21676/2389783X.3219

Recibido en mayo 09 de 2018

Aceptado en junio 20 de 2019

Publicado en línea en noviembre 19 de 2019

Palabras

claves:

infección de la

herida

quirúrgica;

mortalidad;

procedimientos

quirúrgicos;

herida

quirúrgicas

\section{RESUMEN}

Existe evidencia sobre los factores de riesgos asociados a la infección de la herida quirúrgica (IHQ), pero se desconocen estudios que hayan evaluado los factores asociados a la letalidad por la IHQ. Con miras a determinar tales factores, se realizó un estudio de casos y controles anidado a una cohorte de pacientes adultos diagnosticados con IHQ durante el periodo 2015-2016 en un hospital universitario de la región sur de Colombia. Los casos fueron pacientes con IHQ que presentaron letalidad dentro de los 30 días posteriores al inicio de la infección, y los controles correspondieron a aquellos con IHQ que no fallecieron durante dicho lapso. Los pacientes que cumplieron los criterios de selección fueron, en total, 150. Los casos (fallecidos) representaron el $19,3 \%$, quedando el grupo de los controles (vivos) con un $80,7 \%$. Se encontró que, controlando la edad y los días de hospitalización previos al procedimiento quirúrgico, la IHQ tipo órgano espacio es un factor asociado con letalidad en pacientes infectados (OR=2,5 IC 95\% 1,1-6,1). La letalidad en pacientes con IHQ fue elevada y representa un problema de salud pública por las consecuencias que ocasiona al paciente y al sistema de salud.

\section{ABSTRACT}

Keywords: Surgical Wound Infection; Mortality; Surgical Procedures; Surgical Wound.
There is evidence of risk factors associated with surgical site infection (SSI), but there are no studies that have evaluated the factors associated with SSI lethality. We propose a study to determine factors associated with lethality in patients who developed an SSI. A nested case - control study was conducted in adult patients who developed SSI during the period 2015 - 2016 in a university hospital in the southern region of Colombia. The cases were patients with SSI who presented lethality within 30 days after the onset of infection, and the controls who did not die during the 30 days. 150 patients met the selection criteria. The cases (deceased) corresponded to $19.3 \%$, leaving the group of controls (alive) with $80.7 \%$. It was found that controlling the age and days of hospitalization prior to the surgical procedure, organ/space SSI was a factor associated with lethality in infected patients $(\mathrm{OR}=2.595 \% \mathrm{Cl} 1.1-6.1)$. Lethality in patients with SSI was high and represents a public health problem due to the consequences that it causes to the patient and to the health system. 


\section{INTRODUCCIÓN}

Las infecciones asociadas a la atención en salud (IAAS) se consideran un problema de salud pública por la elevada frecuencia con que se producen, la morbilidad y la mortalidad que provocan y -lo más importante- el hecho de que la mayoría son eventos prevenibles ${ }^{1,2}$.

El Centro para el Control y Prevención de Enfermedades (CDC, por sus siglas en inglés) define la IAAS como cualquier condición localizada o sistémica producto de la reacción adversa de agentes infecciosos o sus toxinas y para la que existe evidencia de que no se presentaba o no estaba en incubación en el momento del ingreso del paciente $^{3}$. Se considera que las IAAS se manifiestan después de 48 horas del ingreso o hasta 72 horas después del egreso o atención en salud del paciente ${ }^{4}$.

Una de las infecciones de mayor ocurrencia en las instituciones son las de la herida quirúrgica $(\mathrm{IHQ})^{5}$, que pueden ocurrir hasta 30 días después del procedimiento o un año cuando se involucra componente protésico ${ }^{3}$. A nivel mundial se estima que anualmente ocurren 500.000 $\mathrm{IHQ}$, con una incidencia de tres a cinco infecciones por cada 100 cirugías realizadas ${ }^{6}$. En países desarrollados, entre el $5 \%$ y el $10 \%$ de los pacientes reingresan por infección de la herida quirúrgica, proporción que en algunos países subdesarrollados puede superar el $25 \%{ }^{7}$. Se estima que la IHQ prolonga la estancia hospitalaria en promedio 35 días $^{8}$, incrementa los costos asociados a la atención en salud ${ }^{9,10}$ y contribuye a la generación de microorganismos multirresistentes ${ }^{11,12}$.

Los factores de riesgos para la IHQ se encuentran claramente establecidos. Existen algunos que son propios del paciente, tales como antecedentes personales (diabetes mellitus, hipertensión arterial, insuficiencia renal), el tabaquismo y la edad ${ }^{10}$, y otros se relacionan con la intervención, como el tipo de herida, la intervención quirúrgica y la duración del procedimiento ${ }^{13-}$ 15. También existen recomendaciones basadas en la evidencia para disminuir la incidencia de las infecciones de la herida quirúrgica, como lo es la profilaxis antibiótica ${ }^{16}$.

Se estima que la IHQ se puede prevenir hasta en el 55\% de los $\operatorname{casos}^{17}$, pero una vez sucede el evento, puede ocasionar la letalidad en el $25 \%$ de los infectados ${ }^{18}$. La muerte ocurre principalmente por la condición crítica y la falla orgánica de los pacientes ${ }^{19}$, e incluso el riesgo puede ser mayor en función de la edad avanzada, comorbilidades y el tipo de cirugía o de infección ${ }^{18}$.

La mayoría de los estudios sobre IHQ se han enfocado en identificar los factores de riesgo asociados a la infección, mientras que otros han investigado los factores asociados a la letalidad por la IHQ exclusivamente en pacientes mayores de 75 años $^{18}$. Con esto en mente, se realizó un estudio de casos y controles anidado a una cohorte para establecer los factores asociados a la letalidad en pacientes adultos que desarrollaron una IHQ en una institución prestadora de servicios de salud de tercer nivel de complejidad. La hipótesis del trabajo fue que el tipo de infección es un factor asociado a la letalidad en pacientes con IHQ.

\section{MATERIALES Y MÉTODOS}

\section{Tipo de estudio}

Se llevó a cabo un estudio de casos y controles anidado a una cohorte de pacientes que presentaron $\mathrm{IHQ}$, teniendo en cuenta los criterios del CDC para confirmar la infección ${ }^{3,4}$. Esta se clasificó como IHQ superficial, profunda u órgano espacio según el tipo de tejido involucrado: la superficial involucró piel y tejido subcutáneo; la profunda, fascia y músculo, y la órgano espacio se relacionó con cualquier órgano $u$ otro espacio corporal que se hubiera manipulado durante la cirugía ${ }^{3}$.

La investigación se desarrolló en un hospital universitario de la región sur de Colombia que presta servicios de mediana y alta complejidad, con área de influencia en seis departamentos. La institución cuenta con 390 camas y, en promedio, 38.000 admisiones en el año. Durante el periodo de estudio 2015-2016 se realizaron 10.500 y 11.100 procedimientos quirúrgicos respectivamente.

\section{Población y muestra}

La población estuvo conformada por la cohorte de pacientes que presentaron IHQ según criterios CDC durante el periodo 2015-2016. Se consideraron como casos aquellos con IHQ que fallecieron dentro de los 30 días posteriores al inicio de la infección, mientras que los controles fueron los pacientes con IHQ que no fallecieron durante ese lapso. Para el estudio se seleccionaron todos los usuarios que fueron identificados como caso de IHQ por parte del Comité de Infecciones Intrahosptialarias de la institución de salud. Se excluyeron de la investigación personas que fueron intervenidas quirúrgicamente en otra institución, las que ingresaron a la institución con signos de infección intrahospitalaria y las menores de 18 años.

\section{Variables analizadas}

El desenlace en el estudio fue la letalidad ocurrida durante los 30 días posteriores al inicio de los signos y síntomas de la IHQ. Como variables independientes se analizaron: el sexo, los antecedentes personales, los días de hospitalización previa a la cirugía, el tipo de 
procedimiento, el uso de profilaxis antibiótica, el tipo de herida, el nivel ASA (American Society of Anesthesiologists, por sus siglas en inglés), el riesgo quirúrgico NNIS (National Nosocomial Infection Surveillance, por sus siglas en inglés) y el tipo de IHQ.

Para clasificar el tipo de IHQ se tuvieron en cuenta los criterios del CDC. La infección superficial debió tener al menos uno de los siguientes criterios: drenaje purulento de la incisión superficial o aislamiento de un microorganismo obtenido con todas las medidas asépticas de fluido o tejido de la herida superficial, y diagnóstico de la infección por el equipo quirúrgico durante procedimiento en el quirófano ${ }^{3}$.

La IHQ profunda igualmente debió tener al menos uno de los siguientes criterios: drenaje purulento de la incisión profunda, dehiscencia espontánea o cultivo positivo del drenaje profundo, y absceso u otra evidencia de infección que involucrara tejido profundo y fuera encontrado durante la valoración o reintervención quirúrgica ${ }^{3}$.

Finalmente, la IHQ órgano espacio debió cumplir al menos con uno de los siguientes criterios: drenaje purulento de un dren que estuviera ubicado en un órgano o cavidad, organismo aislado de un cultivo de fluido o tejido de un órgano o cavidad, y absceso u otra evidencia de infección que involucrara algún órgano o cavidad y fuera encontrado durante la valoración o reintervención quirúrgica3.

\section{Procedimientos e instrumentos}

La información se recolectó mediante una revisión documental que incluyó: historia clínica, bases de datos, informes de estadística y reportes quirúrgicos y paraclínicos de los pacientes.

\section{Sesgos}

Para evitar el sesgo de información diferencial, los datos fueron obtenidos por los investigadores con previo entrenamiento y capacitación sobre el instrumento de recolección. También se contó con la supervisión de los investigadores principales durante la captura de la información.

El estudio podría tener un sesgo de centro de referencia ya que se trabajó con pacientes de una institución de salud que presta servicios de mediana y alta complejidad cuya área de influencia es la región surcolombiana. Además, los pacientes que son atendidos por la institución presentan múltiples factores de riesgos, son casos graves y crónicos, y además hacen parte de una población con bajos recursos económicos $(94 \%)^{7,20}$.

\section{Análisis estadístico}

Se compararon los casos y los controles en condiciones basales tales como características sociodemográficas y variables clínicas. Las variables categóricas fueron analizadas mediante proporción, mientras que en las numéricas se comprobó su comportamiento normal a través de la prueba estadística Kolmogórov-Smirnov. Aquellas que no presentaron un comportamiento normal se analizaron con pruebas no paramétricas basadas en la mediana y en su rango.

Para el análisis bivariado se utilizó la prueba de chi cuadrado o test de Fisher (cuando se encontraron valores esperados menores a cinco) para variables categóricas, en tanto que para las numéricas se empleó la prueba tStudent (verificando la igualdad o diferencia de varianzas mediante la prueba Levene) o la prueba U de MannWhitney según la normalidad de la variable. Así mismo, se hizo estimación mediante los intervalos de confianza de las proporciones o las medias con una confiabilidad del $95 \%$.

Se llevó a cabo un análisis por regresión logística binaria donde la variable dependiente fue la letalidad. Como variables independientes se incluyeron las que en el análisis bivariado tuvieron una $p<0,25$, aquellas con importancia clínica y las posibles confusoras. El modelo fue evaluado mediante una prueba de bondad de ajuste y con el coeficiente de determinación. Los resultados fueron expresados en odds ratio (OR), con sus intervalos de confianza del 95\%. Para el análisis estadístico se utilizó el programa R 3.3.3 ${ }^{21}$.

\section{Declaración sobre aspectos éticos}

El protocolo del estudio fue aprobado por el comité de ética de la institución hospitalaria. Se pidió autorización para la revisión de historias clínicas, no se recolectaron datos de información personal de los pacientes, y todos los investigadores firmaron un acuerdo de confidencialidad con la institución. El estudio se clasificó sin riesgo según la Resolución 8430/1993.

\section{RESULTADOS}

Se presentaron 180 casos de infección de la herida quirúrgica (IHQ) durante el periodo 2015-2016. De estos, se excluyeron 23 casos que pertenecían a procedimientos realizados en otras instituciones y 7 de pacientes pediátricos. Se recolectó información de 150 pacientes que cumplieron los criterios de selección. El grupo de los casos (fallecidos) correspondió al 19,3\%, quedando el grupo de los controles (vivos) con un $80,7 \%$ (Tabla 1 ).

Se observó que los casos y los controles fueron homogéneos respecto al sexo, el lugar de residencia, los 
antecedentes personales y la especialidad quirúrgica. La edad fue estadísticamente mayor ( $\dot{x}=61,3$ años IC $95 \%$ $55,4-67,3)$ en el grupo de los casos respecto a los controles. Las comorbilidades de mayor proporción en los pacientes infectados fueron: HTA (19,3\%), DM $(12,7 \%)$ y enfermedad oncológica $(8,7 \%)$, sin diferencia significativa entre los grupos. Las especialidades de cirugía general y ortopedia tuvieron el mayor número de IHQ: $50,7 \%$ y $24,7 \%$ respectivamente (Tabla 1 ).

Tabla 1. Características sociodemográficas y clínicas de pacientes con infección de la herida quirúrgica ( $\mathrm{N}=150)$.

\begin{tabular}{|c|c|c|c|}
\hline Datos & $\begin{array}{c}\text { Casos } \\
\mathbf{N}=\mathbf{2 9} \\
\mathbf{N}(\% ; \text { IC } 95 \%)\end{array}$ & $\begin{array}{c}\begin{array}{c}\text { Controles } \\
N=121\end{array} \\
\text { N (\%; IC 95\%) }\end{array}$ & $\mathbf{P}$ \\
\hline $\begin{array}{c}\text { Sexo } \\
\text { Masculino } \\
\text { Femenino }\end{array}$ & $\begin{array}{l}18(62,1 ; 42,3-79,3) \\
11(37,9 ; 20,7-57,7)\end{array}$ & $\begin{array}{l}65(53,7 ; 44,4-62,8) \\
56(46,3 ; 37,2-55,6)\end{array}$ & 0,41 \\
\hline $\begin{array}{c}\text { Edad (años) } \\
\text { Media (IC95\%) }\end{array}$ & $61,3(55,4-67,3)$ & $47,3(44,1-50,6)$ & $<0,01$ \\
\hline $\begin{array}{c}\text { Lugar de residencia } \\
\text { Rural } \\
\text { Urbano }\end{array}$ & $\begin{array}{c}5(17,2 ; 5,8-35,8) \\
24(82,8 ; 64,2-94,1)\end{array}$ & $\begin{array}{l}30(24,8 ; 17,4-33,5) \\
91(75,2 ; 66,5-82,6)\end{array}$ & 0,38 \\
\hline $\begin{array}{c}\text { Antecedentes personales } \\
\text { DM } \\
\text { EPOC } \\
\text { Secuelas neurológicas } \\
\text { HTA } \\
\text { VIH } \\
\text { Cáncer }\end{array}$ & $\begin{array}{c}3(10,3 ; 2,2-27,2) \\
1(3,4 ; 0,1-17,8) \\
2(6,9 ; 0,8-22,8) \\
9(31,0 ; 15,3-50,8) \\
0 \\
3(10,3 ; 2,2-27,2)\end{array}$ & $\begin{array}{c}16(13,2 ; 7,7-20,6) \\
2(1,6 ; 0,2-5,8) \\
2(1,6 ; 0,2-5,8) \\
20(16,5 ; 10,4-24,4) \\
2(1,6 ; 0,2-5,8) \\
10(8,3 ; 4,0-14,7)\end{array}$ & $\begin{array}{l}0,67 \\
0,69 \\
0,11 \\
0,07 \\
0,48 \\
0,72\end{array}$ \\
\hline $\begin{array}{l}\text { Especialidad quirúrgica } \\
\text { Cirugía general } \\
\text { Ortopedia } \\
\text { Urología } \\
\text { Neurocirugía } \\
\text { Otras especialidades }\end{array}$ & $\begin{array}{c}19(65,5 ; 45,7-82,1) \\
3(10,3 ; 2,2-27,3) \\
0 \\
1(3,4 ; 0,1-17,8) \\
6(20,7 ; 8,0-39,7)\end{array}$ & $\begin{array}{c}57(47,1 ; 38,0-56,4) \\
34(28,1 ; 20,3-37,0) \\
4(3,2 ; 0,9-8,2) \\
8(6,6 ; 2,9-12,6) \\
18(14,9 ; 9,1-22,5)\end{array}$ & $\begin{array}{l}0,07 \\
0,04 \\
\text { N/A } \\
0,51 \\
0,44\end{array}$ \\
\hline
\end{tabular}

1. Prueba t-Student para varianzas iguales.

IC 95\%: intervalos de confianza con una confiabilidad del $95 \%$.

HTA: hipertensión arterial.

DM: diabetes mellitus.

N/A: no aplica.

EPOC: enfermedad pulmonar obstructiva crónica.

Se encontró asociación estadísticamente significativa para letalidad en la IHQ con la estancia prolongada previa a cirugía $(p=0,05)$, la IHQ tipo órgano espacio $(p<0,01)$ y la anestesia general $(p=0,01)$. Se observa que el riesgo de letalidad incrementa de 4,1 en pacientes con IHQ profunda a 7,4 para la infección de órgano espacio (Tabla 2).

Factores como la no administración de profilaxis antibiótica, la duración del procedimiento por más de dos horas y la presencia de alguna complicación quirúrgica como dehiscencia, sepsis o la reexploración quirúrgica se presentaron en mayor proporción en los casos, pero sin significancia estadística.

Se observa que factores como el nivel ASA, el tipo de herida y el riesgo quirúrgico incrementaron el riesgo de letalidad con un nivel de ASA elevado, una herida infectada y un riesgo NNIS mayor, sin significancia estadística (Tabla 2). 
Brayant Andrade-Méndez, Jorge Andrés Ramos-Castañeda, María del Mar Ruiz-García, Edilberto Suaza-Calderón

Tabla 2. Factores asociados a la letalidad en pacientes con infección de la herida quirúrgica.

\begin{tabular}{|c|c|c|c|c|}
\hline Datos & $\begin{array}{c}\text { Casos } \\
\mathrm{N}=29 \\
\mathrm{~N}(\% ; \text { IC } 95 \%) \\
\end{array}$ & $\begin{array}{c}\text { Controles } \\
\mathrm{N}=121 \\
\mathrm{~N}(\% ; \text { IC } 95 \%) \\
\end{array}$ & P2 & $\begin{array}{c}\text { OR } \\
\text { (IC 95\%) }\end{array}$ \\
\hline $\begin{array}{l}\text { Días de hospitalización previos a cirugía } \\
\text { Media (IC 95\%) }\end{array}$ & $11,9(6,1-17,7)$ & $5,9(4,3-7,5)$ & 0,051 & N/A \\
\hline $\begin{array}{l}\text { Tipo de infección } \\
\text { Superficial } \\
\text { Profunda } \\
\text { Órgano espacio } \\
\end{array}$ & $\begin{array}{c}2(6,9 ; 0,8-22,8) \\
14(48,3 ; 29,4-67,5) \\
13(44,8 ; 26,4-64,3) \\
\end{array}$ & $\begin{array}{l}34(28,0 ; 20,3-37,0) \\
58(47,9 ; 38,8-57,2) \\
29(24,0 ; 16,7-32,6)\end{array}$ & $\begin{array}{l}\text { Ref. } \\
0,08 \\
<0,01\end{array}$ & $\begin{array}{c}\text { Ref. } \\
4,1(0,8-39,0) \\
7,4(1,5-73,2)\end{array}$ \\
\hline $\begin{array}{l}\text { Tipo de anestesia } \\
\text { Regional } \\
\text { General }\end{array}$ & $\begin{array}{c}0 \\
29(100 ; 88,0-100)\end{array}$ & $\begin{array}{l}27(22,3 ; 16,4-32,1) \\
94(77,7 ; 69,2-84,7)\end{array}$ & 0,01 & \\
\hline $\begin{array}{l}\text { Profilaxis antibiótica } \\
\text { No }\end{array}$ & $9(31,0 ; 15,3-50,8)$ & $30(24,8 ; 17,4-33,5)$ & 0,49 & $1,39(0,5-3,5)$ \\
\hline $\begin{array}{c}\text { Tipo de herida } \\
\text { Limpia } \\
\text { Limpia contaminada } \\
\text { Contaminada } \\
\text { Infectada }\end{array}$ & $\begin{array}{c}10(34,5 ; 17,9-54,3) \\
5(17,2 ; 5,8-35,8) \\
8(27,6 ; 12,7-47,2) \\
6(20,7 ; 8,0-39,7)\end{array}$ & $\begin{array}{c}42(34,7 ; 26,3-43,9) \\
34(28,1 ; 20,3-37,0) \\
30(24,8 ; 17,4-33,5) \\
15(12,4 ; 7,1-19,6)\end{array}$ & $\begin{array}{c}\text { Ref. } \\
0,4 \\
0,8 \\
0,4\end{array}$ & $\begin{array}{c}\text { Ref. } \\
0,62(0,2-2,0) \\
1,1(0,3-3,6) \\
1,7(0,4-6,2)\end{array}$ \\
\hline $\begin{array}{c}\text { Nivel ASA } \\
\text { I } \\
\text { II } \\
\text { III } \\
\text { IV } \\
\text { V }\end{array}$ & $\begin{array}{c}2(6,9 ; 0,8-22,7) \\
8(27,6 ; 12,7-47,2) \\
14(48,3 ; 29,4-67,5) \\
4(13,8 ; 3,9-31,7) \\
1(3,4 ; 0,1-17,8)\end{array}$ & $\begin{array}{c}22(18,2 ; 11,7-26,2) \\
47(38,8 ; 30,1-48,1) \\
41(33,9 ; 25,5-43,0) \\
9(7,4 ; 3,4-13,6) \\
2(1,6 ; 0,2-5,8)\end{array}$ & $\begin{array}{c}\text { Ref. } \\
0,7 \\
0,1 \\
0,1 \\
0,3\end{array}$ & $\begin{array}{c}\text { Ref. } \\
1,8(0,3-19,4) \\
3,7(0,7-36,5) \\
4,6(0,5-60,2) \\
5,0(0,1-148,9)\end{array}$ \\
\hline $\begin{array}{l}\text { Duración de la cirugía } \\
\text { Más de dos horas }\end{array}$ & $11(37,9 ; 20,7-57,7)$ & $43(35,5 ; 27,0-44,7)$ & 0,81 & $1,1(0,4-2,7)$ \\
\hline $\begin{array}{c}\text { Riesgo quirúrgico NNIS } \\
\text { Bajo } \\
\text { Medio } \\
\text { Medio alto } \\
\text { Alto }\end{array}$ & $\begin{array}{c}1(3,4 ; 0,1-17,8) \\
5(17,2 ; 5,8-35,8) \\
16(55,2 ; 35,7-73,5) \\
7(24,1 ; 10,3-43,5)\end{array}$ & $\begin{array}{c}12(9,9 ; 5,2-16,7) \\
51(42,1 ; 33,2-51,5) \\
43(35,5 ; 27,0-44,7) \\
15(12,4 ; 7,1-19,6)\end{array}$ & $\begin{array}{l}\text { Ref. } \\
1,0 \\
0,2 \\
0,2\end{array}$ & $\begin{array}{c}\text { Ref. } \\
1,2(0,1-60,2) \\
4,4(0,6-202,4) \\
5,4(0,6-272,6)\end{array}$ \\
\hline $\begin{array}{c}\text { Complicación quirúrgica } \\
\text { Dehiscencia } \\
\text { Sepsis } \\
\text { Reexploración quirúrgica }\end{array}$ & $\begin{array}{c}9(31,0 ; 15,3-50,8) \\
17(58,6 ; 38,9-76,5) \\
9(31,0 ; 15,3-50,8)\end{array}$ & $\begin{array}{l}34(28,1 ; 20,3-37,0) \\
55(45,4 ; 36,4-54,7) \\
34(28,1 ; 20,3-37,0)\end{array}$ & $\begin{array}{l}0,75 \\
0,20 \\
0,75\end{array}$ & $\begin{array}{l}1,1(0,4-3,0) \\
1,7(0,7-4,2) \\
1,1(0,4-3,0)\end{array}$ \\
\hline
\end{tabular}

1. Prueba t-Student para varianzas iguales.

2. Prueba de chi cuadrado.

IC $95 \%$ : intervalos de confianza con una confiabilidad del $95 \%$.

N/A: no aplica.

ASA: American Society of Anesthesiologists.

NNIS: National Nosocomial Infection Surveillance.

Al realizar el análisis de regresión logística se encontró que, controlando la edad y los días de hospitalización previos al procedimiento quirúrgico, la IHQ de tipo órgano espacio es un factor asociado a la letalidad en los pacientes infectados. La edad y los días de hospitalización son variables que se relacionan con la IHQ órgano espacio y con la letalidad. Por otra parte, en el análisis de interacción se evidenció que la edad modifica el efecto que produce la IHQ órgano espacio sobre la letalidad en los pacientes (Tabla 3). 
Tabla 3. Infección órgano espacio asociada a la letalidad (análisis de regresión ajustado a edad y días de hospitalización previos a la cirugía).

\begin{tabular}{|l|l|l|}
\hline Datos & P & OR (IC 95\%) Ajustado \\
\hline IHQ órgano espacio & 0,05 & $2,5(1,1-6,1)$ \\
\hline Edad & $<0,01$ & $1,1(1,0-1,1)$ \\
\hline Días de hospitalización previos a la cirugía & 0,05 & $1,1(1,0-1,08)$ \\
\hline Edad * IHQ órgano espacio & 0,02 & N/A \\
\hline
\end{tabular}

\section{DISCUSIÓN}

Producto de la revisión bibliográfica en las principales bases de datos, se puede afirmar que la presente investigación es el primer estudio de casos y controles que analiza los factores asociados a la letalidad en pacientes con IHQ. Se cuenta con evidencia de que los pacientes que desarrollan una IHQ tienen un riesgo hasta 11 veces mayor de morii $^{22,23}$ y de que un $77 \%$ de las muertes en pacientes con IHQ son directamente atribuibles la infección ${ }^{23,24}$.

Los resultados demuestran que la letalidad en pacientes con IHQ fue del $19,3 \%$, inferior al $25,3 \%$ que reportó un estudio sobre mortalidad e infecciones de la herida quirúrgica ${ }^{18}$. Esto se podría deber a que dicha investigación incluyó pacientes mayores de 65 años, lo que se asocia con múltiples comorbilidades y alteraciones funcionales ${ }^{25,26}$. Así mismo, la estancia hospitalaria mayor a tres días previos a la cirugía condiciona la presentación de $\mathrm{IHQ}^{27}$ debido a la colonización por patógenos hospitalarios, que en su mayoría presentan resistencia antibiótica.

Los pacientes adultos mayores que se someten a procedimientos quirúrgicos corren mayor riesgo de morir, producto de enfermedades crónicas como la diabetes mellitus y la hipertensión arterial, que conllevan al deterioro orgánico y del sistema inmune ${ }^{28,29}$. La presente investigación detectó que la edad mayor incrementa el riesgo de letalidad en los pacientes con $\mathrm{IHQ}$, resultado similar a lo reportado por otros estudios $^{25,26}$.

Cirugía general y ortopedia fueron las dos especialidades con mayor número de IHQ, lo que coincide con el tiempo quirúrgico mayor a dos horas, que está directamente relacionado con la presencia de $\mathrm{IHQ}^{30}$. Además, la primera especialidad realiza la mayoría de intervenciones quirúrgicas en el sistema gastrointestinal, que está colonizado por bacterias, y a su vez estos son procedimientos quirúrgicos con duración mayor a dos horas y que, junto con las comorbilidades que presentan los pacientes, incrementan el riesgo de desarrollar infecciones asociadas a la atención en salud.
Otros factores que se asocian al desarrollo de IHQ son el nivel ASA y el tipo de herida, variables que se incluyen dentro del riesgo quirúrgico NNIS ${ }^{31,32}$. Los niveles ASA IV y $\mathrm{V}$, los cuales se asocian con múltiples complicaciones quirúrgicas ya que presentan una tasa de 26,5 por cada 1.000 procedimientos $^{33}$, se encuentran en un $6,7 \%$ de los pacientes con $\mathrm{IHQ}^{34}$ y tienen un $\mathrm{OR}$ de 1,79 en comparación con los pacientes de nivel ASA inferior ${ }^{35}$. Respecto al tipo de herida, las contaminadas e infectadas tienen más de un $20 \%$ de probabilidad de infectarse ${ }^{36}$. En el presente estudio se evidenció que el riesgo de letalidad en pacientes con IHQ podría ser directamente proporcional al riesgo de infección. En otras palabras, el riesgo de letalidad se eleva cuando el nivel ASA, el tipo de herida y el riesgo quirúrgico NNIS aumentan. Esto se debe a la interacción que esos factores de riesgos presentan con el riesgo de infección de la herida quirúrgica ${ }^{31,32}$.

Asimismo, se determinó que la IHQ de tipo órgano espacio es un factor asociado a la letalidad, similar a lo encontrado por Horasan et al ${ }^{18}$. La letalidad en este tipo de infecciones sobreviene por la ubicación en sitios anatómicos complejos y con irrigación comprometida, que hacen dispendiosa la penetración del antibiótico, máxime cuando un gran número de estas infecciones son producidas por gérmenes multirresistentes que presentan una elevada letalidad ${ }^{37,38}$, principalmente debido a la utilización de medicamentos que producen efectos adversos serios ${ }^{39}$.

La letalidad en pacientes con IHQ de tipo órgano espacio se presenta entre un $10,5 \%$ y un $50 \%$, dependiendo de la edad y de la complejidad de la infección ${ }^{40,41}$. En este estudio se encontró que el $31,5 \%$ de los pacientes con ese tipo de infección fallecieron. La elevada letalidad se debe a que la mayoría de los pacientes infectados fueron de cirugía general, especialidad que involucra el sistema gastrointestinal y que ocasiona sepsis severa y falla multiorgánica ${ }^{40}$.

Se considera que la principal limitación del estudio fue que no se midieron factores intrínsecos -como obesidad, tabaquismo, estilos de vida, reportes de paraclínicos como control de la glicemia, albúmina y consumo de medicamentos inmunosupresores - y 
factores extrínsecos - como las medidas de asepsia del personal, ambiente quirúrgico y manejo de la piel-. De igual forma, el hecho de no determinar el agente etiológico de la infección, ni su perfil de resistencia, podría ser otra limitación de la investigación. Este trabajo podría además tener un sesgo de medición en la clasificación ya que fue difícil atribuir la muerte a la infección porque se trataba de pacientes que presentaban múltiples comorbilidades.

En definitiva, se concluye que la IHQ de tipo órgano espacio, la edad y los días previos a la cirugía son factores que se encuentran asociados con la letalidad en los pacientes con IHQ. A su vez, en el contexto de un hospital universitario de alta complejidad, se recomienda reforzar las estrategias de prevención y control de los factores de riesgo para la $\mathrm{IHQ}$ en los momentos pre, intra $y$ posquirúrgico. Ahora bien, una vez que se desarrolle la $\mathrm{IHQ}$, es importante priorizar la atención en pacientes con infección de tipo órgano espacio, edad avanzada y estancia hospitalaria prolongada. Se requieren futuros estudios prospectivos con análisis de supervivencia para evaluar el riesgo de infección instantáneo y por subgrupos de pacientes.

\section{AGRADECIMIENTOS}

Los autores agradecen al Hospital Universitario Hernando Moncaleano Perdomo de Neiva y a su comité de infecciones intrahospitalarias.

\section{DECLARACIÓN SOBRE CONFLICTOS DE INTERESES}

Los autores declaramos no tener ningún conflicto de interés.

\section{CONTRIBUCIÓN DE LOS AUTORES}

Primer autor: trabajo de campo, redacción y elaboración del manuscrito; segundo autor: diseño metodológico, análisis estadístico y elaboración del manuscrito; tercer autor: trabajo de campo y construcción base de datos; cuarto autor: análisis estadístico y elaboración del manuscrito.

\section{REFERENCIAS BIBLIOGRÁFICAS}

1. Instituto Nacional de Salud, Ministerio de Salud. Protocolo de Vigilancia en Salud Pública. Infecciones Asociadas a Dispositivos [internet]. Bogotá: INS; 2014 [citado 20 jun 2018]. Disponible en: http://bvs.minsa.gob.pe/local/MINSA/3450.pdf
2. Organización Mundial de la Salud. Una atención más limpia es una atención más segura [internet]. Washington DC: WHO; 2015 [citado 20 jun 2018]. Disponible en: http://www.who.int/gpsc/background/es/.

3. Horan TC, Andrus $M$, Dudeck MA. CDC/NHSN surveillance definition of health care-associated infection and criteria for specific types of infections in the acute care setting. Am J Infect Control. 2008; 36(5): 309-32. Doi: https://doi.org/10.1016/j.ajic.2008.03.002.

4. Centers for Diseases Control and Prevention. Others. Surgical Site Infection (SSI) Event [internet]. Atlanta: CDC; 2014 [citado 18 may 2018]. Disponible en: http://www.cdc.gov/nhsn/pdfs/pscmanual/9pscssic urrent.pdf.

5. Ministerio de la Protección Social. Informe Nacional de Calidad de la Atención en Salud, 2015 [internet]. Bogotá DC; 2015. [citado 20 jun 2018]. Disponible en: https://www.minsalud.gov.co/sites/rid/Lists/Bibliot ecaDigital/RIDE/DE/DIJ/informe-nal-calidadatencion-salud-2015.pdf.

6. Allegranzi B, Nejad SB, Combescure C, Graafmans W, Attar $\mathrm{H}$, Donaldson $\mathrm{L}$, et al. Burden of endemic health-care-associated infection in developing countries: systematic review and meta-analysis. The Lancet. 2011; 377(9761): 228-41. Doi: http://doi.org/10.1016/S0140-6736(10)61458-4.

7. Fierro JD, Naranjo MA, Cabrera C, Ramos JA. Caracterización epidemiológica de las infecciones asociadas a la atención en salud en una IPS privada. Neiva 2013. Rev Fac Salud-RFS. 2016; 7(2): 29-34. Doi: http://doi.org/10.25054/rfs.v7i2.952.

8. González L, Toledo S, Parra Y, Santofimio D, Osorio J, Ramos J. Infecciones intrahospitalarias en servicios de medicina interna y cirugía del Hospital Universitario de Neiva, 2012. RFS. 2015; 5(2): 27-33. Doi: http://doi.org/10.25054/rfs.v5i2.139.

9. Lemos EV, Fernando P, Alvis N, Quevedo E, Einarson $T R$, Castañeda $C$, et al. Costos en pacientes con infección por Acinetobacter baumannii en Colombia. Infectio [revista en la internet]. 2013 [citado 20 jun 2018]; 17(4): 185-192. Disponible en: http://www.scielo.org.co/scielo.php?script=sci_artt ext\&pid=S0123-93922013000400004.

10. Laloto TL, Gemeda DH, Abdella SH. Incidence and predictors of surgical site infection in Ethiopia: prospective cohort. BMC Infect Dis. 2017; 17(1): 119. Doi: http://dx.doi.org/10.1186/s12879-016-2167-x. 
11. Hawser SP, Bouchillon SK, Lascols $C$, Hackel M, Hoban DJ, Badal RE, et al. Susceptibility of Klebsiella pneumoniae isolates from intra-abdominal infections and molecular characterization of ertapenem-resistant isolates. Antimicrob Agents Chemother. 2011; 55(8): 3917-21. Doi: http://dx.doi.org/10.1128/AAC.00070-11.

12. Magill SS, Edwards JR, Bamberg W, Beldavs ZG, Dumyati G, Kainer MA, et al. Multistate pointprevalence survey of health care-associated infections. N Engl J Med. 2014; 370(13): 1198-208. Doi: http://dx.doi.org/10.1056/NEJMoa1306801.

13. Isik O, Kaya E, Dundar HZ, Sarkut P. Surgical Site Infection: Re-assessment of the Risk Factors. Chirurgia [revista en la internet]. 2015 [citado 18 may 2018]; 110(5): 457-461.. Disponible en: http://revistachirurgia.ro/pdfs/2015-5-457.pdf.

14. Khan M, Rooh-ul-Muqim ZM, Khalil J, Salman M. Influence of ASA score and Charlson Comorbidity Index on the surgical site infection rates. J Coll Physicians Surg Pak. 2010; 20(506): e509. Doi: http://dx.doi.org/08.2010/JCPSP.506509.

15. Chen LF, Kaye D. Current use for old antibacterial agents: polymyxins, rifamycins, and aminoglycosides. Infect Dis Clin North Am. 2009; 23(4): $\quad$ 1053-75. Doi: http://dx.doi.org/10.1016/j.idc.2009.06.004.

16. Allegranzi $B$, Zayed B, Bischoff $P$, Kubilay $N Z$, Jonge $S$ de, Vries $F$ de, et al. New WHO recommendations on intraoperative and postoperative measures for surgical site infection prevention: an evidence-based global perspective. Lancet Infect Dis. 2016; 3099(16): 30402-9. Doi: http://dx.doi.org/10.1016/S14733099(16)30402-9.

17. Umscheid CA, Mitchell MD, Doshi JA, Agarwal R, Williams K, Brennan PJ. Estimating the Proportion of Healthcare-Associated Infections That Are Reasonably Preventable and the Related Mortality and Costs. Infect Control Amp Hosp Epidemiol. 2011; 32(2): 101-14. Doi: http://dx.doi.org/ $10.1086 / 657912$.

18. Horasan ES, Dağ A, Ersoz G, Kaya A. Surgical site infections and mortality in elderly patients. Med Mal Infect. 2013; 43(10): 417-22. Doi: http://dx.doi.org/10.1016/j.medmal.2013.07.009.

19. Ferreira FL, Bota DP, Bross A, Mélot C, Vincent J-L. Serial evaluation of the SOFA score to predict outcome in critically ill patients. Jama. 2001; 286(14): $1754-8$.

Doi: http://dx.doi.org/10.1001/jama.286.14.1754.
20. González L, et al. Infecciones intrahospitalarias en servicios de medicina interna y cirugía del Hospital Universitario de Neiva, 2012. Rev Fac Salud-RFS. 2015; 5(2): 27-33. Doi: http://doi.org/10.25054/rfs.v5i2.139.

21. R Core Team. R: A language and environment for statistical computing. R Foundation for Statistical Computing, Vienna, Austria. 2008 [internet] [citado 12 sep 2017]. Disponible en: http://softlibre.unizar.es/manuales/aplicaciones/r/f ullrefman.pdf.

22. Engemann JJ, Carmeli Y, Cosgrove SE, Fowler VG, Bronstein MZ, Trivette SL, et al. Adverse clinical and economic outcomes attributable to methicillin resistance among patients with Staphylococcus aureus surgical site infection. Clin Infect Dis. 2003; 36(5): 592-8. Doi: http://doi.org /10.1086/367653.

23. Anderson DJ, Podgorny K, Berríos-Torres SI, Bratzler DW, Dellinger EP, Greene L, et al. Strategies to prevent surgical site infections in acute care hospitals: 2014 update. Infect Control Hosp Epidemiol. 2014; 35(S2): S66-88. Doi: http://doi.org/10.1086/676022.

24. Mangram AJ, Horan TC, Pearson ML, Silver LC, Jarvis WR, Committee HICPA, et al. Guideline for prevention of surgical site infection, 1999. Am J Infect Control. 1999; 27(2): 97-134. Doi: http://doi.org/10.1016/S0196-6553(99)70088-X.

25. Raymond DP, Pelletier SJ, Crabtree TD, Schulman AM. Surgical infection and the aging population/Discussion. Am Surg [revista en la internet]. 2001 [citado 12 sep 2017]; 67(9): 827. Disponible en: https://www.ncbi.nlm.nih.gov/pubmed/11565758.

26. Kaye KS, Anderson DJ, Sloane R, Chen LF, Choi Y, Link $K$, et al. The Effect of Surgical Site Infection on Older Operative Patients. J Am Geriatr Soc. 2009; 57(1): 4654. Doi: http://doi.org/10.1111/j.15325415.2008.02053.x.

27. Londoño Á, Morales J, Murilla M. Características epidemiológicas y factores de riesgo relacionados con la infección en el sitio operatorio en procedimientos de cirugía general. Rev Chil Cir. 2011; 63(6): 559-565. Doi: http://doi.org/10.4067/S071840262011000600003.

28. Kaye KS, Schmit K, Pieper C, Sloane R, Caughlan KF, Sexton DJ, et al. The effect of increasing age on the risk of surgical site infection. J Infect Dis. 2005; 191(7): 1056-62. Doi: http://doi.org/10.1086/428626. 
29. Despaigne Alba I, Rodríguez Fernández Z, Pascual Bestard M, Lozada Prado GA, Mustelier Ferrer HL. Consideraciones actuales sobre las infecciones posoperatorias. Medisan [revista en la internet]. 2013 [citado 18 may 2018]; 17(4): 686-707. Disponible en: http://scielo.sld.cu/pdf/san/v17n4/san16413.pdf.

30. Fajardo Rodríguez HA, Quemba Gordillo J, Eslava Schmalbach J. Escalas de predicción e infección de sitio quirúrgico en 15625 cirugías 2001-2003. Rev Salud Pública [revista en la internet]. 2005 [citado 10 sep 2017]; 7(1): 89-98. Disponible en: http://www.scielo.org.co/pdf/rsap/v7n1/v7n1a7.pd f.

31. National Nosocomial Infections Surveillance System. National Nosocomial Infections Surveillance (NNIS) system report, data summary from January 1992 through June 2004, issued October 2004. Am J Infect Control. 2004; 32: 470-85. Doi: http://doi.org/10.1016/j.ajic.2004.10.001.

32. Emori TG, Culver DH, Horan TC, Jarvis WR, White JW, Olson DR, et al. National nosocomial infections surveillance system (NNIS): Description of surveillance methods. Am J Infect Control. 1991; 19(1): 19-35. Doi: http://doi.org/10.1016/01966553(91)90157-8.

33. Daabiss M. American Society of Anaesthesiologists physical status classification. Indian J Anaesth. 2011; 55(2): 111-5. Doi: http://doi.org/10.4103/00195049.79879 .

34. Tang R, Chen HH, Wang YL, Changchien CR, Chen J-S, Hsu K-C, et al. Risk Factors For Surgical Site Infection After Elective Resection of the Colon and Rectum: A Single-Center Prospective Study of 2,809 Consecutive Patients. Ann Surg. 2001; 234(2): 181-9. Disponible en: http://www.ncbi.nlm.nih.gov/pmc/articles/PMC142 2004/.

35. Ridgeway $S$, Wilson J, Charlet A, Kafatos G, Pearson $A$, Coello R. Infection of the surgical site after arthroplasty of the hip. Bone Jt J. 2005; 87-B(6): 844-
50. Doi: http://doi.org/10.1302/0301620X.87B6.15121.

36. López Tagle D, Hernández Ferrer M, Saldivar Arias T, Sotolongo Hernández T, Valdés Dupeyrón 0 . Infección de la herida quirúrgica: Aspectos epidemiológicos. Rev Cuba Med Mil [revista en la internet]. 2007 [citado 22 mar 2018]; 36(2): 0-0. Disponible en: http://scielo.sld.cu/pdf/mil/v36n2/mil08207.pdf.

37. Clara L, Stern L, Barcán L, Marchetti M, Greco G. Mediastinitis post-quirúrgica: Estudio caso - control. Rev Chil Infectol. 2002; 19(1): 37-48. Doi: http://dx.doi.org/10.4067/S071610182002000100006.

38. Ramos-Castañeda JA, Ruano-Ravina A, BarbosaLorenzo R, Paillier-Gonzalez JE, Saldaña-Campos JC, Salinas DF, et al. Mortality due to KPC carbapenemase-producing Klebsiella pneumoniae infections: Systematic review and meta-analysis: Mortality due to KPC Klebsiella pneumoniae infections. J Infect. 2018; 76(5): 438-448. Doi: http://doi.org/10.1016/j.jinf.2018.02.007.

39. Osorio J, Barreto J, Samboni CF, Cándelo LA, Álvarez LC, Benavidez S, et al. Factores asociados a nefrotoxicidad por polimixina $B$ en un hospital universitario de Neiva, Colombia. 2011-2015. Rev Chil Infectol. 2017; 34(1): 7-13. Doi: http://dx.doi.org/10.4067/S071610182017000100001.

40. Rodríguez Fernández Z, Wendy G, Tamayo M, Elmer M, García R, Ibrahim L, et al. Mortalidad y reintervenciones en cirugía general. Medisan [revista en la internet]. 2012 [citado 20 jun 2018]; 16(11): 1676-89. Disponible en: http://scielo.sld.cu/pdf/san/v16n11/san041112.pdf.

41. Rodríguez Fernández $Z$, Fernández López $O$, Romero García LI. Factores asociados a la mortalidad por infecciones posoperatorias. Rev Cuba Cir [revistas de internet]. 2016 [citado 16 may 2018]; 55(2). Disponible en: http//scielo.sld.cu/pdf/cir/v55n2/cir04216.pdf. 